\title{
A Nursing Assessment Tool for Adults With Fecal Incontinence
}

\section{Christine Norton, MA, RN, and Sonya Chelvanayagam, MSc, RN}

\begin{abstract}
Fecal incontinence affects slightly more than $1 \%$ of community-dwelling adults. This article describes an assessment format, with a research basis when available, that has been developed in a specialist nursing clinic in the United Kingdom. The focus is on how to obtain the most useful information from the patient to plan appropriate nursing interventions. A subsequent article will describe the biofeedback program developed as part of a package of care to meet individual needs of persons with fecal incontinence.
\end{abstract}

\section{Introduction}

Fecal incontinence is a common health care problem, affecting more than $1 \%$ of the adult population. ${ }^{1}$ It is a particularly embarrassing symptom that is socially unacceptable, yet many with the problem do not seek professional help. ${ }^{2}$ Fecal incontinence has a major negative impact on physical and psychological health and lifestyle, with severe social restrictions in many instances. ${ }^{3}$

Fecal incontinence has multiple possible causes, the most common being obstetric trauma. ${ }^{4}$ Other causes include congenital, traumatic, or iatrogenic sphincter damage; intestinal hypermotility resulting in diarrhea; neurologic disease; local anorectal pathology; and, in immobile individuals, fecal impaction with overflow soiling. ${ }^{5,6}$ Recent advances in investigation techniques, particularly anorectal manometry and endoanal ultrasound, enable accurate characterization of structural or functional causes of fecal incontinence in individual patients. Nevertheless, treatment options are limited for many who primarily rely on surgery or constipating drugs to alter stool consistency and diminish the risk of subsequent incontinence.

Fecal continence is a complex function that involves multiple modulatory mechanisms that influence stool consistency, intestinal motility, rectal vault storage, and anal sphincter function. Therefore, when managing a patient with fecal incontinence, it is essential to define what combination of these factors contributes to soiling. Although previous authors have stressed the importance of assessment $^{7}$ and provided general guidelines for management, ${ }^{8,9}$ our search of the literature found no tool adequate for the assessment of fecal incontinence in a population of independent, communitydwelling adults. We decided that an essential first step when working with patients with fecal incontinence at our hospital specializing in colorectal dysfunction was to define important areas of assessment and pertinent demographic data. The checklist we developed to assess these variables is provided in Figure 1. The main focus is fecal incontinence, although many patients have additional bowel problems, and some questions were designed to explore these aspects of gastrointestinal function. 
This assessment instrument is not intended to measure formally such important psychosocial aspects of fecal incontinence as coping or quality of life; instead, it provides baseline information intended to direct the formulation of a plan of care. A combination of empiric (clinical) experience and literature review was used to design the instrument. We found this combination necessary because research evidence is not available at this stage to substantiate every aspect of the assessment. Completion of the instrument requires approximately 20 to 30 minutes. This article will review the domains covered in the initial assessment and provide a rationale for the clinical relevance of each item

\section{THE ASSESSMENT INTERVIEW}

When managing a patient with a socially and personally sensitive condition such as fecal incontinence, it is important to build a relationship of trust so that symptoms and problems can be openly and frankly discussed. This assessment also requires the patient and nurse to establish a vocabulary of words that are mutually understood and acceptable. Even though all of our patients have been referred specifically for therapy (usually biofeedback) for fecal incontinence, many express embarrassment and find it difficult to report symptoms. The experience seems to be similar to that described for urinary incontinence, another taboo subject that is not openly discussed, even with partners or family. ${ }^{10}$ The need for a common vocabulary is compounded because some patients are unaware of medical terms such as "defecation" or even "stool" or "anus"; but they are reluctant to use what they perceive as slang terms when discussing fecal incontinence with a professional. The wording we use for each item is indicated by italics. When reading this article and considering applying this instrument to your practice, it should be remembered that we designed the questions for an English population, and colloquial expressions will require adaptation for use in other patient populations. The essential point is to find a vocabulary that is mutually understood by both nurse and patient.

Chief Symptom

What bothers you about your bowels/what is the main problem with your bowels? This item was designed to help the nurse understand the patient's perspective on the problem and identify which symp toms are most bothersome. We have found that the symptom identified by the patient as most bothersome may be quite different from the primary symptom documented by the referring physician. Circumstances surrounding the onset of the problem can give important clues as to causation. Sometimes a causative event can be clearly identified, such as onset of symptoms immediately after traumatic childbirth or a hemorrhoidectomy. At other times the patient may not have made a link between potential contributing factors and the onset of fecal incontinence. In these cases, probing questions about a change in medication or onset of menopause may help to identify contributing factors. Many people cope with fecal incontinence for years without seeking professional help. ${ }^{2}$ In these patients, we find it useful to explorwhat triggered consultation. Have the symptoms worsened? Has the ability to cope with symptoms been compromised?

\section{Bowel Elimination Patterns}

How often do you move/open your bowels? There is a common misconception among the general population that normal bowel habit is once per day. In fact, function varies between 1 to 3 times a day to once in 3 days. ${ }^{11} \mathrm{~A}$ minority of the total population, approximately $40 \%$ of men and $33 \%$ of 
women, move their bowels once on a daily basis. ${ }^{12}$ Instead, most are irregular in the frequency of bowel movements, with women of childbearing age (25-49 years) being the most irregular. One third of young women move their bowels less than once per day and $1 \%$ do so less than once per week. A variable bowel habit is a classic feature of irritable bowel syndrome (IBS). ${ }^{13,14}$

Knowledge of this variability is important when assessing a patient's bowel elimination patterns because of common misconceptions about the frequency of bowel movements and constipation. The term constipation is interpreted differently by various persons. ${ }^{15}$ Infrequent bowel motions, provided the stool is easy to pass and not hardened, does not meet clinical criteria of constipation and it is not a cause for concern or justification for intervention. Conversely, some very constipated people can produce a stool several times per day, but only at the expense of long periods of straining on the toilet. There is, in the general population, no age-related decrease in the actual frequency of defecation, although there seems to be an increase with age in self-report of constipation and an increase in laxative use. ${ }^{16}$ The use of a laxative does not necessarily imply a diagnosis of constipation.

A recent change in the frequency of bowel motions should be noted because it may indicate an underlying disease or malignancy. Any recent unexplained change in bowel habit in a patient older than 40 years old should be investigated by barium enema radiograph or colonoscopy. Although fecal incontinence is rarely the sole presenting symptom for intestinal cancer, up to $25 \%$ of patients with cancer note occasional bouts of fecal soiling. ${ }^{17}$

A diary of bowel actions for a week often provides useful baseline information (Figure 2). This diary is routinely sent to patients with their appointment letter, and very few fail to bring it, correctly completed, to the first consultation.

\section{Stool Consistency}

What is your stool (bowel movement) like? Is it loose, soft but formed, hard or hard pellets? Does this vary? Careful assessment of stool consistency with particular attention to reported changes in consistency or color also provides important information in the evaluation of fecal incontinence. For example, patients with IBS or inflammatory bowel disease are particularly prone to variations in stool consistency. When the patient has difficulty in describing the stool, a visual prompt may be helpful (Figure 3). ${ }^{12}$ This type of scale is useful because patients have been found to be generally accurate when assessing their own stool consistency. ${ }^{18}$ When asked to respond to this 7-point visual scale for stool consistency, types 3 or 4 represent the most usual selections. Nevertheless, only 56\% of women describe these types as normal, whereas $61 \%$ of men describe them as normal. Loose stool is significant because it increases the risk for both passive and urge fecal incontinence; a pelletlike stool consistency is important because it may indicate slow colonic transit and constipation

\section{Fecal Incontinence}

A particularly sensitive query is required when asking patients about fecal incontinence because many people are reluctant to admit stool soiling, and it may be difficult to identify terms expressing this sign that are mutually understood and acceptable. Even people consulting a gastroenterologist often refrain from volunteering the symptom of fecal incontinence. In one study, less than half of the patients admitting to incontinence on a questionnaire had told their doctor about it. ${ }^{2}$ In addition 
to reluctance in identifying the symptom of fecal incontinence, it can be difficult to assess the severity of soiling because many people severely restrict their lifestyle, thus limiting the risk of incontinence. As a result, fecal incontinence may occur infrequently, even though it represents a major concern. Many patients also find it difficult to estimate the amount of stool lost during an incontinent episode. We have found it particularly helpful to question patients about the 2 major types of fecal incontinence: urge or passive.

\section{Urgency and Urge Incontinence}

When you need to open/move your bowels, do you need to rush to get to the toilet? How longcan you hold on for? When the rectum fills, the internal anal sphincter relaxes reflexively to enable "sampling" of the rectal contents by the sensitive nerve endings within the dentate line in the upper anal canal (Figure 4). With normal bowel control, defecation can be deferred for long periods of time because the urge to defecate is opposed by voluntary contraction of the striated external anal sphincter muscle, ${ }^{19}$ which generates sufficient pressure to prevent immediate stool expulsion and to return the stool to the rectum (Figure 5). Retrograde peristalsis may even remove the stool away from the anal sphincter and back into the sigmoid colon in some cases. ${ }^{20}$ If bowel control is normal, the urge diminishes, and the patient is typically able to defer defecation for up to several hours. Indeed, it has been shown that voluntarily ignoring the call to defecate can reduce the frequency of defecation by $50 \%$ and slow colonic transit. ${ }^{21}$

In contrast, a reduced squeeze pressure and an inability to sustain a submaximal contraction of the external sphincter has been found to correlate with the symptom of urgency. ${ }^{22,23}$ Some patients with fecal incontinence generate high amplitude colonic pressure waves (up to $500 \mathrm{~cm}$ water pressure). ${ }^{20}$ Without an adequate sphincter response to oppose this high-pressure bolus contraction, resistance to the expulsion of stool is diminished and urge incontinence is likely. In addition, patients who have diarrhea, such as those with inflammatory bowel disease, are at a particularly high risk of severe urgency and urge incontinence from colonic peristalsis opposing sphincter function and to the loose consistency of the rectal contents.

Do you ever fail to reach the toilet in time and have a bowel accident? Urge incontinence often reflects a weakness or defect in the external anal sphincter, ${ }^{24,25}$ and obstetric trauma is the most common cause of sphincter damage. ${ }^{26}$ Urgency may become a particular problem because of a fear of future accidents for the individual who already has had an episode of fecal incontinence. As a result, the patient may respond immediately to any urge to defecate in an attempt to prevent incontinent episodes. This rapid response may create a vicious cycle so that any bowel sensation is interpreted as likely to lead to incontinence. Because of this fear, a sensation of rectal urgency generates intense anxiety, or even panic, which exacerbates the sense of urgency. When a patient reports rectal urgency, it is important to establish how frequently urge incontinence actually occurs and whether restrictions in activities are from actual or feared soiling.

\section{Passive Incontinence}

Do you have any leakage from your bottom of which you are unaware? Is this liquid or solid? Does this occur at any time or only after you have opened/moved your bowels? The smooth muscle of the internal anal sphincter is responsible for up to $80 \%$ of resting tone in the anal canal, ${ }^{5}$ and passive soiling is associated with internal anal sphincter damage detected by ultrasound. ${ }^{24,25} \mathrm{~A}$ weak or 
disrupted internal sphincter is incapable of completely closing the anal canal. If the stool is loose or soft, some may remain in the anal canal and ooze out after defecation. Incompetence of the internal anal sphincter is often the result of inadvertent surgical damage (particularly after hemorrhoidectomy or sphincterotomy) or idiopathic smooth muscle degeneration. ${ }^{27}$ Patients with internal anal sphincter incompetence have difficulty cleaning the anus after defecation and experience subsequent soiling, which may persist for several hours. Others have passive soiling at unpredictable times and in the absence of any awareness of stool soiling. In still others, soiling is provoked or exacerbated by physical exertion such as walking or running. Passive loss of solid stool or loss of copious amounts of mucus may result from rectal prolapse.

\section{Flatus}

Can you control wind? Are you able to tell the difference between wind and the need to empty your bowels? Although the concept of "wind" is a common expression for the passage of flatus among English patients, nurses from the United States may prefer to ask patients if they are able to control the passage of "gas" from the bowels. In addition, it is important to determine whether patients are able to distinguish between the passage of flatus and stool. This sensory deficit may occur after iatrogenic or traumatic damage to the sensory nerves involved in the "sampling" reflex previously described. Incontinence of flatus may appear a trivial symptom, but many people who are unable to control the passage of flatus experience embarrassment in work, social, or intimate situations

\section{Pain}

Do you have pain associated with open-

ing/moving your bowels? Does it occur before

moving your bowels and is relieved by opening

your bowels, or is it a pain as you actually pass

a stool?

Abdominal pain in association with

altered stool consistency, frequency, or

bloating in the absence of organic patholo-

gy is diagnostic of IBS,

14

which is common

among patients with fecal incontinence.

Pain with the urge to defecate may occur in

these patients, rendering it difficult to 
defer defecation. They may describe a colicky or spasmodic "stabbing" pain. In contrast, the occurrence of anal pain with defecation is often caused by hemorrhoids or an anal fissure, which are also common among patients with fecal incontinence. Chronically constipated people also tend to report abdominal discomfort; it is usually characterized by a feeling of bloating. Constipation or constipation-predominant IBS is also associated with abdominal bloating; we have observed that some women report the need for two sets of clothes because of this bloating. They have a normal wardrobe, but they also own clothes that are 1 to 2 sizes larger for periods when their bowels have not moved for several days.

\section{Blood and Mucus}

Do you pass any blood or mucus when your bowels move/open?

The passage of blood or stool during defecation occurs with a number of conditions. For example, streaks of bright red blood may occur in patients with hemorrhoids or an anal fissure. The passage of fresh (bright red) or older (darker red) 
blood may also occur in patients with an underlying malignancy. The presence of blood and mucus in an individual with a 